\title{
Optimum Design for Sparse FDA-MIMO Automotive Radar
}

\author{
Saeid Sedighi, Student Member, IEEE, Bhavani Shankar, Senior Member, IEEE, Kumar Vijay Mishra, Senior \\ Member, IEEE, and Björn Ottersten, Fellow, IEEE \\ Interdisciplinary Centre for Security, Reliability and Trust (SnT), University of Luxembourg Email: \\ \{saeid.sedighi@, bhavani.shankar@, kumar-mishra@ext., bjorn.ottersten@\}uni.lu
}

\begin{abstract}
Automotive radars usually employ multiple-input multiple-output (MIMO) antenna arrays to achieve high azimuthal resolution with fewer elements than a phased array. Despite this advantage, hardware costs and desired radar size limits the usage of more antennas in the array. Similar trade-off is encountered while attempting to achieve high range resolution which is limited by the signal bandwidth. However, nowadays given the demand for spectrum from communications services, wide bandwidth is not readily available. To address these issues, we propose a sparse variant of Frequency Diverse Array MIMO (FDA-MIMO) radar which enjoys the benefits of both FDA and MIMO techniques, including fewer elements, decoupling, and efficient joint estimation of target parameters. We then employ the Cramér-Rao bound for angle and range estimation as a performance metric to design the optimal antenna placement and carrier frequency offsets for the transmit waveforms. Numerical experiments suggest that the performance of sparse FDA-MIMO radar is very close to the conventional FDA-MIMO despite $50 \%$ reduction in the bandwidth and antenna elements.
\end{abstract}

Index Terms-Automotive radar, Cramér-Rao bound, frequency diverse array, MIMO radar, optimization.

\section{INTRODUCTION}

Automotive radars are an increasingly essential sensor in self-driving cars and Advanced Driver Assistant Systems (ADASs) [1], [2]. These sensors are responsible for detecting vehicular targets and obstacles in harsh weather and low visibility [3], [4]. The ability to correctly ascertain these objects is severely hampered by the limited range and angular resolution of the radar [5]. While the radar range resolution is restricted by its bandwidth, the angular resolution is directly proportional to the array aperture and the number of receive channels [6]. Increasing any one of these design parameters - bandwidth, array aperture, receive channels - leads to high cost and large radar footprint on the vehicle. In this paper, we focus on the problem of reducing the bandwidth and antennas in automotive radar arrays while maintaining the performance.

The antennas in automotive radars commonly employ multiple-input-multiple-output (MIMO) arrays which use several transmit (Tx) and receive $(\mathrm{Rx})$ antennas [7], [8]. In a colocated MIMO radar [9], [10], the antennas are located so close that the radar cross-section (RCS) appears

The work of Saeid Seidghi is supported by the Luxembourg National Research Fund (FNR) under the ACCORDION project (reference number 11228830). The work of the other authors is partially funded by ERC AGNOSTIC (Grant ID: 742648) and the FNR under the BRIDGES project AWARDS identical to all Tx-Rx antenna pairs. Unlike a phased array, the MIMO transmitters emit mutually orthogonal signals. The receivers then exploit the phase difference in the signals leading to an improved angular resolution [11], [12] and high parameter identifiability [13]. The improved azimuth resolution is achieved with fewer Tx-Rx antennas than a phased array operating with the same aperture [14].

Recently, frequency diverse array (FDA) technique [15] has received significant research interest [16]. The FDA radar introduces a tiny frequency offset (FO) across the array elements. The result is a beampattern that is jointly dependent on range, angle, and time [17]. In contrast, a phased array has only angle-dependent beampattern [18]. Such a radar is able to efficiently localize targets in a joint range-angle domain [19]. By introducing nulls in its pattern, the radar is capable of suppressing range-dependent interference and clutter. Recent works [20] have also shown that further enhancement in FDA ranging accuracy is possible through coherent integration of multiple FDA pulses in slow-time domain. Finally, the auto-scanning feature of FDA radar implies that phase shifters are not required to steer the beam as in a conventional phased array radar [21].

Given the advantages of MIMO and FDA over conventional phased arrays, there is immense interest in hybridizing these two technologies in a FDA-MIMO radar [19], [22], which combines the joint range-angle-Doppler processing of FDA with the decoupling and low footprint advantages of MIMO. In the automotive radar scenario, conventional FDA-MIMO array still suffers from large hardware costs. Further, introduction of the FOs requires additional bandwidth. To this end, in this paper, we propose a sparse FDA-MIMO which utilizes a small fraction of the conventional FDA-MIMO spectrum and aperture without serious performance loss.

Sparsity-based techniques have a rich heritage of research in radar signal processing [23]. Most of these methods leverage the presence of a limited number of targets in the region of interest to reduce the resources such as bandwidth, sapling rates and array size. The signal recovery and target detection is then achieved with compressive sensing (CS) [24]. A large body of literature exists on the spatial, temporal, and Doppler domain applications of CS to reduce, respectively, the array size, bandwidth, and pulses in a sparsity-based radar. [25]-[28].

In this paper, our goal is to find an optimal antenna placement and FOs in a sparse FDA-MIMO such that an 
accurate estimation of target's direction-of-arrival (DoA) and range is guaranteed. Since we use fewer antennas and limited FOs in a sparse FDA-MIMO than its conventional counterpart, peak performance is traded off. The exact reduction could be determined by the tolerance to such performance loss. Once antenna placement and FOs are determined, the signal recovery is performed via CS. In this work, we focus only on the joint antenna and FO selection problem for a colocated FDA-MIMO [19], [22], [29]. A few works on FO selection in FDA arrays have previously employed random [30] and co-prime [31] offfsets. However, their application in a hybrid FDA-MIMO remains unexamined. We consider the Cramér-Rao bound (CRB) as the performance criterion in our joint design problem which we cast as a non-convex problem. Later, we relax this to a semi-definite program (SDP) and propose an iterative solution to the problem. Contrary to earlier works, our proposed FDA-MIMO automotive radar offers lower footprint with enhanced capabilities derived from both FDA and MIMO arrays.

The rest of the paper is organized as follows. Section II describes the system model. The problem formulation is given in Section III] Section IV] provides the proposed algorithm for designing the antenna placements and the set of carrier frequencies. The simulation results and related discussions are included in Section V] Finally, Section VI concludes the paper.

Throughout this paper, we refer the vectors and matrices by lower- and upper-case bold-face, respectively. The superscripts $*, T$ and $H$ denote the conjugate, transpose and Hermitian operations, respectively. $\|\mathbf{a}\|_{1}$ stands for the $\ell_{1}$-norm of $\mathbf{a}$. The cardinality of the set $\mathbb{A}$ is represented by $|\mathbb{A}| .[\mathbf{A}]_{i, j}$ and $[\mathbf{a}]_{i}$ indicate the $(i, j)^{\text {th }}$ and $i^{\text {th }}$ entry of $\mathbf{A}$ and $\mathbf{a}$, respectively. The $M \times M$ identity matrix is denoted by $\mathbf{I}_{M} . \mathbb{E}\{$.$\} stands$ for the statistical expectation. $\otimes$ represents Kronecker product. $\operatorname{vec}(\mathbf{A})=\left[\begin{array}{llll}\mathbf{a}_{1}^{T} & \mathbf{a}_{2}^{T} & \cdots & \mathbf{a}_{n}^{T}\end{array}\right]^{T}$ denotes the vectorization operation.

\section{SySTEM MOdEL}

Consider a sparse colocated FDA-MIMO radar equipped with $M$ transmit and $L$ receive antennas. Let the operating wavelength of the radar be $\lambda$. The $m^{\text {th }}$ transmit and $l^{\text {th }}$ receive antennas are situated, respectively, at $\frac{\lambda}{2} d_{m}$ and $\frac{\lambda}{2} u_{l}$ with $d_{m}$, $u_{l} \in \mathbb{S} \doteq\{0,2, \cdots, R-1\}$. Further, the $m^{\text {th }}$ transmit antenna emits narrowband pulses of frequency $f_{m}=f_{c}+a_{m} \triangle f$, where $a_{m} \in \mathbb{H} \doteq\{0,1, \cdots, N-1\}$. Here, $f_{c}$ denotes the reference carrier frequency and $\triangle f$ is the carrier frequency increment unit across the transmit antennas. Illustrative examples of the transceiver array configuration and the FDMA transmission scheme are shown in Figs. 1 and 2, respectively. Accordingly, the signal transmitted by the $m^{\text {th }}$ transmit antenna is

$$
s_{m}(t)=\sqrt{P_{t}} h(t) e^{-j 2 \pi\left(f_{c}+a_{m} \triangle f\right) t}, \quad 0 \leq t \leq T,
$$

where $P_{t}$ is the transmit power and $h(t)$ denotes unit-energy waveform, i.e., $\int_{0}^{T} h(t) h^{*}(t)=1$ with bandwidth $B_{h}$.

The target scene is composed of $Q$ stationary point-targets, with the $q^{\text {th }}$ target parameterized by the following parameters unknown to the radar: the reflection coefficient $\xi_{q}$ which is directly proportional to the target's radar cross section (RCS)

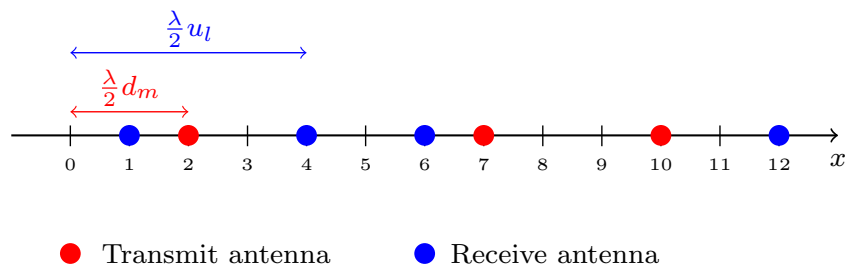

Fig. 1. Illustration of the transceiver array geometry with $M=3$ transmit antennas and $L=4$ receive antenna.

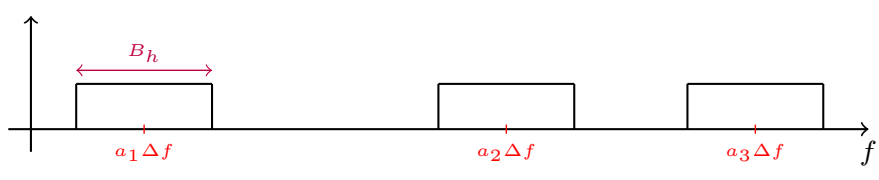

Fig. 2. Illustration of the sparse FDMA transmission scheme with $M=3$ carrier frequencies.

of the target and propagation factors; the range $r_{q}$ of the target from the radar; azimuth angle $\theta_{q}$; and the radial velocity $v_{q}$. We make following assumptions about the target and radar parameters:

A1 "Non-overlapping bandwidths": The carrier frequency increment unit $\triangle f>B_{h}$, so that the intervals $\left[a_{m} \triangle f-\frac{B_{h}}{2}, a_{m} \triangle f+\frac{B_{h}}{2}\right]$ do not overlap for all $m$. This assumption enables orthogonal transmissions for FDA-MIMO Radar.

A2 "Negligible phase-frequency distortion": The maximum carrier frequency increment across transmit antennas is negligible compared to the reference carrier frequency, i.e., $\max _{m} \frac{a_{m} \Delta f}{f_{c}} \ll 1$. This assumption is valid for millimeter wave radar where, for example, $f_{c}=79 \mathrm{GHz}$ while $\max a_{m} \triangle f$ is, at most, of the order of a few MHz.

A3 "Small aperture": This allows $\xi_{q}$ 's and $\theta_{q}$ 's to be constant over the array elements.

A4 "Far ranges": Targets are in the far-field, i.e., $r_{q} \gg \frac{\lambda}{2} R$.

Assuming that the propagation channel is non-dispersive and $h(t)$ is narrowband, the back-scattered signal from the $Q$ targets at the $l^{\text {th }}$ receive antenna is

$$
\begin{aligned}
& x_{l}(t)=\sum_{q=1}^{Q} \sum_{m=1}^{M} \sqrt{P_{t}} \xi_{q} h\left(t-\frac{2 r_{q}}{c}\right) \\
& \times e^{-j 2 \pi\left(f_{c}+a_{m} \triangle f\right)\left(t-\tau_{q, m}-\tau_{q, l}\right)},
\end{aligned}
$$

where $\tau_{q, m}=\frac{r_{q}+\frac{\lambda d_{m}}{2} \sin \theta_{q}}{c}$ and $\tau_{q, l}=\frac{r_{q}+\frac{\lambda u_{l}}{2} \sin \theta_{q}}{c}$ are the time delay between the $m^{\text {th }} / l^{\text {th }}$ transmit/receive antenna and the $q^{\text {th }}$ target. The signal $x_{l}(t)$ is downconverted to baseband and sampled at rate $B_{h}$. Taking a $K$-point Discrete Fourier Transform (DFT), where $K=T B_{h}$, of the sampled signal yields

$$
\begin{aligned}
X_{l}(k) & =\sum_{q=1}^{Q} \sum_{m=1}^{M} \alpha_{q} H\left(2 \pi\left(\frac{k}{T}-a_{m} \Delta f\right)\right) \\
& \times e^{-j 4 \pi\left(a_{m} \Delta f+\frac{B_{h}}{T} k\right) \frac{r_{q}}{c}} e^{-j \pi\left(d_{m}+u_{l}\right) \sin \theta_{q}},
\end{aligned}
$$


where $0 \leq k \leq K-1, \alpha_{q}=\sqrt{P_{t}} \xi_{q} e^{j 4 \pi\left(\frac{r_{q}}{\lambda_{c}}\right)}, \lambda_{c}=\frac{c}{f_{c}}$, and $H(\omega)=\int_{-\infty}^{\infty} h_{m}(t) e^{-j \omega t} d t$ is the continuous-time Fourier transform (CTFT) of $h(t)$. Exploiting A1, it is possible to separate $X_{l}(k)$ into components corresponding to each transmit antenna. Passing $X_{l}(k)$ through a filter bank, which consists of $M$ filters with central frequencies $a_{m} \triangle f$ and bandwidth $B_{h}$, and then multiplying with $H^{*}\left(2 \pi\left(\frac{k}{T}-a_{m} \Delta f\right)\right)$ produces

$$
\begin{aligned}
& \begin{aligned}
\tilde{X}_{m, l}(k)= & \left|H\left(2 \pi\left(\frac{k}{T}-a_{m} \Delta f\right)\right)\right|^{2} \sum_{q=1}^{Q} \alpha_{q} e^{-j 4 \pi\left(a_{m} \Delta f+\right.} \\
& \times e^{-j \pi\left(d_{m}+u_{l}\right) \sin \theta_{q}} \cdot
\end{aligned} \\
& \text { Let } y_{m, l}(k)=\frac{1}{\mid H\left(\left.2 \pi\left(\frac{k}{T}-a_{m} \Delta f\right)\right|^{2}\right.} \tilde{X}_{l, m}(k), \text { then } \\
& y_{m, l}(k)=\sum_{q=1}^{Q} \alpha_{q} e^{-j 4 \pi\left(a_{m} \Delta f+\frac{B_{h}}{T} k\right) \frac{r_{q}}{c}} e^{-j \pi\left(d_{m}+u_{l}\right) \sin \theta_{q}}
\end{aligned}
$$

In the presence of additive noise, the filtered signal is

$$
z_{m, l}(k)=y_{m, l}(k)+e_{m, l}(k),
$$

where $e_{m, l}(k)$ is the noise trail at the output of the $m^{\text {th }}$ filter of $l^{\text {th }}$ receiver. The $e_{m, l}(k), \forall l, m, k$ are modeled as identical and independently distributed (i.i.d.) zero-mean Gaussian random variables with variance $\sigma^{2}$. Stacking all the measurements in a $M L K \times 1$ vector, we obtain the measurements as

$$
\mathbf{z}=\mathbf{y}+\mathbf{e} \in^{M L K \times 1} .
$$

Using this system model, our goal is to design antenna placement and the set of carrier frequencies for the considered sparse FDA-MIMO radar such that the performance loss becomes as small as possible.

\section{CRB-BASED OptimiZATION FRAMEWORK}

The CRB provides a lower bound on the mean-squared-error (MSE) matrix of any mean-unbiased estimator in a non-Bayesian setting [32]. We, therefore, use it as a design metric for finding the optimal antenna placement and the set of carrier frequencies for the transmit waveforms. However, the CRB depends on the unknown target parameters, which makes the desired optimization problem highly complicated. To alleviate this problem, we consider the CRB for two targets which has been shown to only depend on the difference between the parameters of two targets. This attribute of two-target CRB is very helpful in simplifying the desired optimization problem while delivering a good performance. Indeed, two-target CRB is capable of controlling for both the mainlobe width and the sidelobe level of the ambiguity function [25].

Define $\mathbb{D}=\left\{d_{1}, d_{2}, \cdots, d_{M}\right\}, \mathbb{U}=\left\{u_{1}, u_{2} \cdots, u_{L}\right\}$ and $\mathbb{A}=\left\{a_{1}, a_{2}, \cdots, a_{M}\right\}$ Further, let $\boldsymbol{\rho}_{q}=\left[r_{q}, \sin \theta_{q}\right]^{T}$ denote the vector of the unknown parameter of the $q^{\text {th }}$ target and $\rho=$ $\left[\boldsymbol{\rho}_{1}^{T}, \cdots, \boldsymbol{\rho}_{Q}^{T}\right]^{T}$ be the vector of all the unknown parameters. The CRB is the inverse of the Fisher Information Matrix (FIM) [32]

$$
\begin{aligned}
& \mathbf{F}(\boldsymbol{\rho}, \mathbb{D}, \mathbb{U}, \mathbb{A})= \\
& \mathrm{E}\left\{\frac{\partial \ln f(\mathbf{z} \mid \boldsymbol{\rho}, \mathbb{D}, \mathbb{U}, \mathbb{A})}{\partial \boldsymbol{\rho}}\left(\frac{\partial \ln f(\mathbf{z} \mid \boldsymbol{\rho}, \mathbb{D}, \mathbb{D}, \mathbb{A})}{\partial \boldsymbol{\rho}}\right)^{H}\right\},
\end{aligned}
$$

where $f(\mathbf{z} \mid \boldsymbol{\rho}, \mathbb{D}, \mathbb{U}, \mathbb{A})$ denotes the conditional probability density function of $\mathbf{z}$. Since the noise is i.i.d., we obtain

$\ln f(\mathbf{y} \mid \boldsymbol{\rho}, \mathbb{D}, \mathbb{U}, \mathbb{A})=\sum_{m=1}^{M} \sum_{l=1}^{L} \sum_{k=0}^{K-1} \ln f\left(z_{m, l}(k) \mid \boldsymbol{\rho}, d_{m}, u_{l}, a_{m}\right)$,

leading to [32]

$$
\mathbf{F}(\boldsymbol{\rho}, \mathbb{D}, \mathbb{U}, \mathbb{A})=\frac{4}{\sigma^{2}} \sum_{m=1}^{M} \sum_{l=1}^{L} \sum_{k=0}^{K-1} \frac{\partial y_{m, l}(k)}{\partial \boldsymbol{\rho}}\left(\frac{\partial y_{m, l}(k)}{\partial \boldsymbol{\rho}}\right)^{H} .
$$

For two-target CRB, the derivative of $y_{m, l}(k)$ with respect to $\rho$ is

$$
\frac{\partial y_{m, l}(k)}{\partial \boldsymbol{\rho}}=-\mathrm{j} \pi\left[\begin{array}{c}
\frac{4}{c}\left(a_{m} \Delta f+\frac{B_{h}}{T} k\right) y_{m, l}^{1}(k) \\
\left(d_{m}+u_{l}\right) y_{m, l}^{1}(k) \\
\frac{4}{c}\left(a_{m} \triangle f+\frac{B_{h}}{T} k\right) y_{m, l}^{2}(k) \\
\left(d_{m}+u_{l}\right) y_{m, l}^{2}(k)
\end{array}\right],
$$

where $y_{m, l}^{q}(k)=\alpha_{q} e^{-j 4 \pi\left(a_{m} \triangle f+\frac{B_{h}}{T} k\right) \frac{r_{q}}{c}} e^{-j \pi\left(d_{m}+u_{l}\right) \sin \theta_{q}}$ Consequently, the two-target CRB is the inverse of

$$
\begin{aligned}
\mathbf{F}(\boldsymbol{\rho}, \mathbb{D}, \mathbb{U}, \mathbb{A})= & \frac{4 \pi^{2}}{\sigma^{2}} \sum_{m=1}^{M} \sum_{l=1}^{L} \sum_{k=0}^{K-1} \\
& \check{\mathbf{F}}_{k}\left(\boldsymbol{\rho}, d_{m}, u_{l}, a_{m}\right) \otimes \overline{\mathbf{F}}_{k}\left(d_{m}, u_{l}, a_{m}\right),
\end{aligned}
$$

where

$$
\begin{aligned}
& \check{\mathbf{F}}_{k}\left(\boldsymbol{\rho}, d_{m}, u_{l}, a_{m}\right)= \\
& {\left[\begin{array}{cc}
\left|\alpha_{1}\right|^{2} & \alpha_{1} \alpha_{2}^{*} \alpha_{q} e^{-j 4 \pi\left(a_{m} \triangle f+\right.} \\
\alpha_{1}^{*} \alpha_{2} e^{-j 4 \pi\left(a_{m} \triangle f+\frac{B_{h}}{T} k\right) \frac{r_{2}-r_{1}}{c}} e^{-j \pi\left(d_{m}+u_{l}\right)\left(\sin \theta_{2}-\sin \theta_{1}\right)} & \\
\overline{\mathbf{F}}_{k}\left(d_{m}, u_{l}, a_{m}\right)=\left[\begin{array}{cc}
\frac{16}{c^{2}}\left(a_{m} \triangle f+\frac{B_{h}}{T} k\right)^{2} & \frac{4}{c}\left(a_{m} \triangle f+\frac{B_{h}}{T} k\right)\left(d_{m}+u_{l}\right) \\
\frac{4}{c}\left(a_{m} \triangle f+\frac{B_{h}}{T} k\right)\left(d_{m}+u_{l}\right) & \left(d_{m}+u_{l}\right)^{2}
\end{array}\right] .
\end{array}\right.}
\end{aligned}
$$

It readily follows that the FIM is a function of the difference between the DoAs and ranges of the targets.
Hence, $\mathbf{F}(\boldsymbol{\rho}, \mathbb{D}, \mathbb{U}, \mathbb{A})=\mathbf{F}(\Delta \boldsymbol{\rho}, \mathbb{D}, \mathbb{U}, \mathbb{A})$ where $\Delta \rho=\left[r_{1}-\right.$ $\left.r_{2}, \sin \theta_{1}-\sin \theta_{2}\right]^{T}$. We discretize the two dimensional space 
$\mathcal{P}$ of possible values of $\Delta \rho$. Thereafter, we cast the joint design of antenna placement and the set of carrier frequencies for the transmit waveforms as the following optimization problem

$$
\begin{array}{ll}
\min _{\mathbb{D}, \mathbb{U}, \mathbb{A}} \max _{\Delta \boldsymbol{\rho} \in \mathcal{P}} & f\left(\mathbf{F}^{-1}(\Delta \boldsymbol{\rho}, \mathbb{D}, \mathbb{U}, \mathbb{A})\right) \\
\text { subject to } & |\mathbb{D}| \leq M, \\
& d_{m} \in \mathbb{S}, \quad d_{i} \neq d_{j}, \quad \forall i, j, \\
& |\mathbb{U}| \leq L, \\
& u_{l} \in \mathbb{S}, \quad u_{i} \neq u_{j}, \quad \forall i, j, \\
& |\mathbb{A}| \leq M, \\
& a_{m} \in \mathbb{H}, \quad a_{i} \neq a_{j}, \quad \forall i, j,
\end{array}
$$

where $f($.$) is a proper scalar function of the CRB. A$ multi-parameter CRB-based optimization in [25] suggested various choices for the function $f($.$) . In this paper, we$ employ the E-optimality criteria, wherein $f($.$) is the maximum$ eigenvalue of $\mathbf{F}^{-1}(\boldsymbol{\rho}, \mathbb{D}, \mathbb{U}, \mathbb{A})$. Since $(15)$ is non-convex, we propose to employ its convex relaxation, as discussed in the next section.

\section{Antenna Placement and Carrier Frequencies}

Define the selection vector $\mathbf{b} \in\{0,1\}^{R \times 1}$ and the selection matrix $\mathbf{T} \in\{0,1\}^{R \times N}$ as

$$
\begin{gathered}
{[\mathbf{b}]_{l}= \begin{cases}1, & \text { if } l-1 \in \mathbb{U}, \\
0, & \text { otherwise, }\end{cases} } \\
{[\mathbf{T}]_{m, n}= \begin{cases}1, & \text { if } m-1 \in \mathbb{T} \text { and } n-1 \in \mathbb{A}, \\
0, & \text { otherwise. }\end{cases} }
\end{gathered}
$$

Then, the FIM in (12) becomes

$$
\begin{aligned}
\mathbf{F}(\Delta \boldsymbol{\rho}, \mathbf{b}, \mathbf{T})= & \frac{4 \pi^{2}}{\sigma^{2}} \sum_{m=1}^{R} \sum_{n=1}^{N} \sum_{l=1}^{R} \sum_{k=0}^{K-1}[\mathbf{b}]_{l}[\mathbf{T}]_{m, n} \\
& \times\left(\check{\mathbf{F}}_{m, n, l, k} \otimes \overline{\mathbf{F}}_{m, n, l, k}\right),
\end{aligned}
$$

where

$$
\begin{aligned}
& \check{\mathbf{F}}_{m, n, l, k}=\left[\begin{array}{cc}
\left|\alpha_{1}\right|^{2} & \left.\alpha_{1} \alpha_{2}^{*} \alpha_{q} e^{-j 4 \pi\left(n \triangle f+\frac{B_{h}}{T} k\right) \frac{r_{1}-r_{2}}{c}} e^{-j \pi(m+l)\left(\sin \theta_{1}-\sin \theta_{2}\right.}\right) \\
\alpha_{1}^{*} \alpha_{2} e^{-j 4 \pi\left(n \triangle f+\frac{B_{h}}{T} k\right) \frac{r_{2}-r_{1}}{c}} e^{-j \pi(m+l)\left(\sin \theta_{2}-\sin \theta_{1}\right)} & \left|\alpha_{2}\right|^{2}
\end{array}\right], \\
& \overline{\mathbf{F}}_{m, n, l, k}=\left[\begin{array}{cc}
\frac{16}{c^{2}}\left(n \triangle f+\frac{B_{h}}{T} k\right)^{2} & \frac{4}{c}\left(n \triangle f+\frac{B_{h}}{T} k\right)(m+l) \\
\frac{4}{c}\left(n \triangle f+\frac{B_{h}}{T} k\right)(m+l) & (m+l)^{2}
\end{array}\right] .
\end{aligned}
$$

Accordingly, the optimization problem 15 is recast as

$$
\begin{array}{cl}
\min _{\mathbf{b}, \mathbf{T}} \max _{\Delta \boldsymbol{\rho} \in \mathcal{P}} & \lambda_{\max }\left(\mathbf{F}^{-1}(\Delta \boldsymbol{\rho}, \mathbf{b}, \mathbf{T})\right) \\
\text { subject to } & \mathbf{b} \in\{0,1\}^{R \times 1} \\
& \mathbf{T} \in\{0,1\}^{R \times N} \\
& \|\mathbf{b}\|_{1} \leq L \\
& \|\operatorname{vec}(\mathbf{D})\|_{1} \leq 2 M
\end{array}
$$

We relax the min-max optimization problem above as

$$
\begin{array}{ll}
\max _{\mathbf{b}, \mathbf{T}, \gamma} & \gamma \\
\text { subject to } & \mathbf{F}(\Delta \boldsymbol{\rho}, \mathbf{b}, \mathbf{T}) \succeq \gamma \mathbf{I}_{4}, \quad \forall \Delta \boldsymbol{\rho} \in \mathcal{P}, \\
& \|\mathbf{b}\|_{1} \leq L, \\
& \|\operatorname{vec}(\mathbf{D})\|_{1} \leq 2 M \\
& \mathbf{b} \in\{0,1\}^{R \times 1} \\
& \mathbf{T} \in\{0,1\}^{R \times N}
\end{array}
$$

The relaxation in (22) encourages the eigenvalues of $\mathbf{F}^{-1}(\Delta \boldsymbol{\rho}, \mathbf{b}, \mathbf{T})$ to be as small as possible. However, 22$)$ is still non-convex because of the products of unknowns (see (18)). To address this, introduce $\mathbf{g}=\left[\mathbf{b}^{T}, \operatorname{vec}^{T}(\mathbf{T})\right]^{T} \in$ $\{0,1\}^{R(N+1) \times 1}$ and $\mathbf{G}=\mathbf{g g}^{T} \in\{0,1\}^{R(N+1) \times R(N+1)}$, so that 22 is recast as

$$
\begin{array}{ll}
\max _{\mathbf{g}, \mathbf{G}, \gamma} & \gamma \\
\text { subject to } & \mathbf{F}(\Delta \boldsymbol{\rho}, \mathbf{G}) \succeq \gamma \mathbf{I}_{4}, \quad \forall \Delta \boldsymbol{\rho} \in \mathcal{P}, \\
& \mathbf{G}=\mathbf{g g}^{T}, \\
& \sum_{j=1}^{R}[\mathbf{g}]_{i} \leq L, \\
& \sum_{j=R+1)}^{R(N+1)}[\mathbf{g}]_{i} \leq 2 M \\
& \mathbf{g} \in[0,1]^{R(N+1) \times 1},
\end{array}
$$

where we have also relaxed the binary constraint on the elements of $\mathbf{g}$ by allowing its elements to be within $[0,1]$. This problem is a SDP with an equality constraint, which is non-convex. From [33, Lemma 1], it follows that the equality constraint can be replaced with a rank constraint on a semi-definite matrix. Therefore, the equivalent optimization problem is

$$
\begin{array}{ll}
\max _{\mathbf{g}, \mathbf{G}, \gamma} & \gamma \\
\text { subject to } & \mathbf{F}(\Delta \boldsymbol{\rho}, \mathbf{G}) \succeq \gamma \mathbf{I}_{4}, \quad \forall \Delta \boldsymbol{\rho} \in \mathcal{P}, \\
& {\left[\begin{array}{cc}
1 & \mathbf{g} \\
\mathbf{g}^{T} & \mathbf{G}
\end{array}\right] \succeq \mathbf{0},} \\
& \operatorname{rank}\left(\left[\begin{array}{cc}
1 & \mathbf{g} \\
\mathbf{g}^{T} & \mathbf{G}
\end{array}\right]\right) \leq 1, \\
& \sum_{j=1}^{R}[\mathbf{g}]_{i} \leq L, \\
& \sum_{j=R+1}^{R(N+1)}[\mathbf{g}]_{i} \leq 2 M, \\
& \mathbf{g} \in[0,1]^{R(N+1) \times 1}
\end{array}
$$


We solve this problem iteratively using Algorithm 11, where the sequential problem at the $i^{\text {th }}$ iteration is

$$
\begin{array}{ll}
\max _{\mathbf{g}_{i}, \mathbf{G}_{i}, \gamma_{i}} & \gamma_{i}+w_{i} e_{i} \\
\text { subject to } & \mathbf{F}\left(\Delta \boldsymbol{\rho}, \mathbf{G}_{i}\right) \succeq \gamma_{i} \mathbf{I}_{4}, \quad \forall \Delta \boldsymbol{\rho} \in \mathcal{P}, \\
& {\left[\begin{array}{cc}
1 & \mathbf{g}_{i} \\
\mathbf{g}_{i}^{T} & \mathbf{G}_{i}
\end{array}\right] \succeq \mathbf{0},} \\
& e_{i} \mathbf{I}_{R(N+1)-1}-\mathbf{V}_{i-1}^{H}\left[\begin{array}{cc}
1 & \mathbf{g}_{i} \\
\mathbf{g}_{i}^{T} & \mathbf{G}_{i}
\end{array}\right] \mathbf{V}_{i-1} \succeq \mathbf{0}, \\
& \sum_{j=1}^{R}\left[\mathbf{g}_{i}\right]_{j} \leq L, \\
& \sum_{j=R+1}^{R(N+1)}\left[\mathbf{g}_{i}\right]_{j} \leq 2 M, \\
& \mathbf{g}_{i} \in[0,1]^{R(N+1) \times 1} \\
& e_{i} \leq e_{i-1},
\end{array}
$$

where $\mathbf{V}_{k-1}$ contains the eigenvectors corresponding to the $R(N+1)-1$ smallest eigenvalues of $\left[\begin{array}{cc}1 & \mathbf{g}_{i} \\ \mathbf{g}_{i}^{T} & \mathbf{G}_{i}\end{array}\right]$ obtained at the previous iteration. Indeed, the optimization problem that needs to be worked out at each step of Algorithm 1 is an SDP for which efficient solvers exist. Further, $\mathbf{V}_{0}$ is obtained through a relaxed solution of 24] in which the rank constraint is dropped.

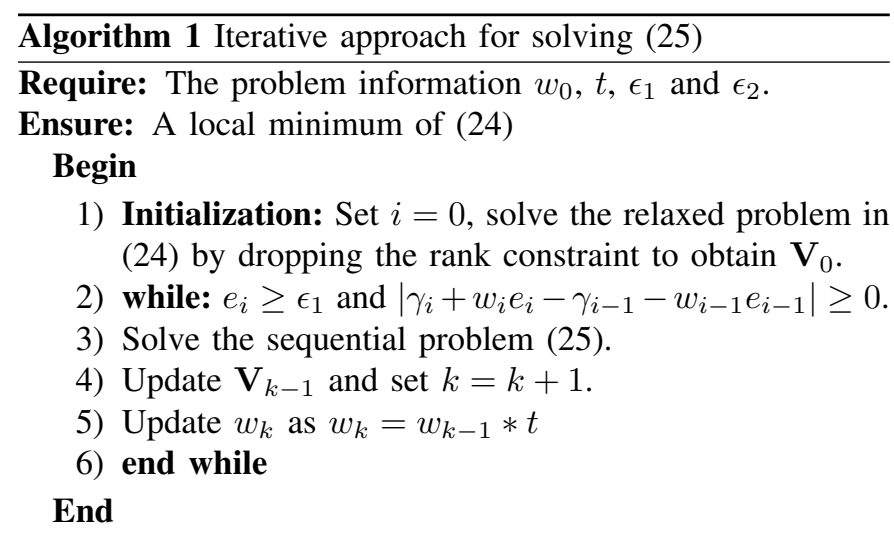

\section{NUMERICAL EXPERIMENTS}

We compared the performance of the proposed sparse FDA-MIMO Radar with the conventional FDA-MIMO Radar through numerical experiments. We set the following parameters for the sparse FDA-MIMO radar: $f_{c}=79 \mathrm{GHz}$, $\Delta f=150 \mathrm{MHz}, B_{h}=120 \mathrm{MHz}, R=12, N=6, M=3$, $L=3$. The conventional FDA-MIMO radar had similar design parameters except that both the number of transmit and receive antennas as well as the bandwidth were twice that of sparse FDA-MIMO.

Figure 3 shows the two-target $\mathrm{CRB}$ of the sparse and conventional FDA-MIMO radar. We observe that even though the sparse FDA-MIMO radar uses half of transmit and receive antennas and half of the bandwidth, its performance loss is not significant when compared with the conventional FDA-MIMO radar. This indicates the advantage of using the proposed design for the sparse FDA-MIMO radar.

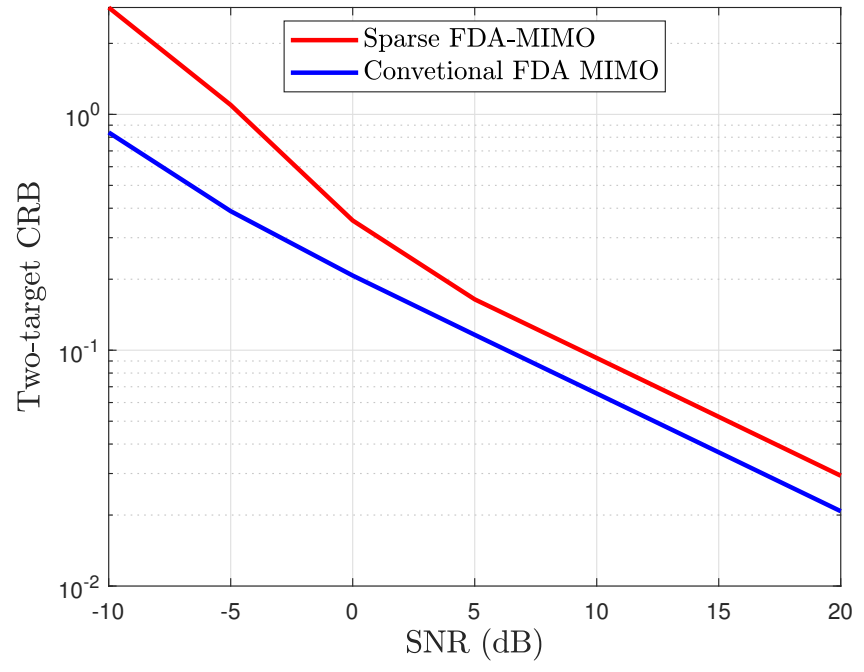

Fig. 3. Maximum $\theta_{2}$ versus SNR for the sparse and conventional FDA-MIMO radar with $\mathrm{SNR}=0 \mathrm{~dB}$ and $K=5$.

\section{SUMMARY}

Small footprint of automotive radar arrays is highly desirable. To this end, we proposed an approach for designing antenna placement and the portion of spectrum for a sparse FDA-MIMO radar such that it guarantees a desired DoA and range estimation accuracy. We considered two-target CRB as the performance criterion for such a joint design. We cast the resulting non-convex optimization problem as a SDP and proposed an iterative algorithm to attain the design solution. We demonstrated that the proposed radar system is capable of reducing the hardware costs and bandwidth while retaining a reasonable estimation performance.

\section{REFERENCES}

[1] K. V. Mishra, M. R. Bhavani Shankar, V. Koivunen, B. Ottersten, and S. A. Vorobyov, "Toward millimeter wave joint radar communications: A signal processing perspective," IEEE Signal Processing Magazine, vol. 36, pp. 100-114, 2019.

[2] G. Duggal, S. Vishwakarma, K. V. Mishra, and S. S. Ram, "Doppler-resilient 802.11ad-based ultra-short range automotive radar," arXiv preprint arXiv:1902.01306, 2019.

[3] S. M. Patole, M. Torlak, D. Wang, and M. Ali, "Automotive radars: A review of signal processing techniques," IEEE Signal Processing Magazine, vol. 34, no. 2, pp. 22-35, 2017.

[4] Z. Slavik and K. V. Mishra, "Phenomenological modeling of millimeter-wave automotive radar," in 2019 URSI Asia-Pacific Radio Science Conference, 2019, pp. 1-4.

[5] F. Engels, P. Heidenreich, A. M. Zoubir, F. K. Jondral, and M. Wintermantel, "Advances in automotive radar: A framework on computationally efficient high-resolution frequency estimation," IEEE Signal Processing Magazine, vol. 34, no. 2, pp. 36-46, 2017.

[6] M. A. Richards, Fundamentals of radar signal processing. McGraw-Hill, 2005

[7] E. Fishler, A. Haimovich, R. Blum, D. Chizhik, L. Cimini, and R. Valenzuela, "MIMO radar: An idea whose time has come," in IEEE Radar Conference, 2004, pp. 71-78.

[8] M. S. Davis, G. A. Showman, and A. D. Lanterman, "Coherent MIMO radar: The phased array and orthogonal waveforms," IEEE Aerospace and Electronic Systems Magazine, vol. 29, no. 8, pp. 76-91, 2014.

[9] J. Li and P. Stoica, "MIMO radar with colocated antennas," IEEE Signal Processing Magazine, vol. 24, no. 5, pp. 106-114, 2007.

[10] W. Khan, I. M. Qureshi, and K. Sultan, "Ambiguity function of phased-MIMO radar with colocated antennas and its properties," IEEE Geoscience and Remote Sensing Letters, vol. 11, no. 7, pp. 1220-1224, 2014. 
[11] H. Godrich, A. M. Haimovich, and R. S. Blum, "Target localization accuracy gain in MIMO radar-based systems," IEEE Transactions on Information Theory, vol. 56, no. 6, pp. 2783-2803, 2010.

[12] R. Boyer, "Performance bounds and angular resolution limit for the moving colocated MIMO radar," IEEE Transactions on Signal Processing, vol. 59, no. 4, pp. 1539-1552, 2011.

[13] A. M. Haimovich, R. S. Blum, and L. J. Cimini, "MIMO radar with widely separated antennas," IEEE Signal Processing Magazine, vol. 25, no. 1, pp. 116-129, 2008

[14] K. V. Mishra, Y. C. Eldar, E. Shoshan, M. Namer, and M. Meltsin, "A cognitive sub-nyquist mimo radar prototype," IEEE Transactions on Aerospace and Electronic Systems, 2019, in press.

[15] P. Antonik, M. C. Wicks, H. D. Griffiths, and C. J. Baker, "Frequency diverse array radars," in IEEE Conference on Radar, 2006, pp. 1-3.

[16] A. Basit, W. Khan, S. Khan, and I. M. Qureshi, "Development of frequency diverse array radar technology: A review," IET Radar, Sonar \& Navigation, vol. 12, no. 2, pp. 165-175, 2017.

[17] A. Basit, I. M. Qureshi, W. Khan, S. ur Rehman, and M. M. Khan, "Beam pattern synthesis for an FDA radar with Hamming window-based nonuniform frequency offset," IEEE Antennas and Wireless Propagation Letters, vol. 16, pp. 2283-2286, 2017.

[18] K. V. Mishra, I. Kahane, A. Kaufmann, and Y. C. Eldar, "High spatial resolution radar using thinned arrays," in IEEE Radar Conference, 2017, pp. 1119-1124.

[19] J. Xu, G. Liao, S. Zhu, L. Huang, and H. C. So, "Joint range and angle estimation using MIMO radar with frequency diverse array," IEEE Transactions on Signal Processing, vol. 63, no. 13, pp. 3396-3410, 2015.

[20] R. Gui, W.-Q. Wang, Y. Pan, and J. Xu, "Cognitive target tracking via angle-range-doppler estimation with transmit subaperturing FDA radar," IEEE Journal of Selected Topics in Signal Processing, vol. 12, no. 1, pp. 76-89, 2018.

[21] W.-Q. Wang, "Overview of frequency diverse array in radar and navigation applications," IET Radar, Sonar \& Navigation, vol. 10, no. 6, pp. 1001-1012, 2016.

[22] J. Xiong, W.-Q. Wang, and K. Gao, "FDA-MIMO radar range-angle estimation: CRLB, MSE, and resolution analysis," IEEE Transactions on Aerospace and Electronic Systems, vol. 54, no. 1, pp. 284-294, 2017.
[23] K. V. Mishra and Y. C. Eldar, "Sub-Nyquist radar: Principles and prototypes," in Compressed Sensing in Radar Signal Processing, A. D. Maio, Y. C. Eldar, and A. Haimovich, Eds. Cambridge University Press, 2019, pp. 1-48.

[24] J. H. Ender, "On compressive sensing applied to radar," Signal Processing, vol. 90, no. 5, pp. 1402-1414, 2010.

[25] E. Tohidi, M. Radmard, S. Karbasi, H. Behroozi, and M. Nayebi, "Compressive sensing in MTI processing," in IEEE International Workshop on Compressed Sensing Theory and its Applications to Radar, Sonar and Remote Sensing, 2015, pp. 189-193.

[26] H. Godrich, A. P. Petropulu, and H. V. Poor, "Sensor selection in distributed multiple-radar architectures for localization: A knapsack problem formulation," IEEE Transactions on Signal Processing, vol. 60, no. 1, pp. 247-260, 2011.

[27] S. Joshi and S. Boyd, "Sensor selection via convex optimization," IEEE Transactions on Signal Processing, vol. 57, no. 2, pp. 451-462, 2008.

[28] V. Roy, S. P. Chepuri, and G. Leus, "Sparsity-enforcing sensor selection for DOA estimation," in IEEE International Workshop on Computational Advances in Multi-Sensor Adaptive Processing, 2013, pp. 340-343.

[29] M. Secmen, S. Demir, A. Hizal, and T. Eker, "Frequency diverse array antenna with periodic time modulated pattern in range and angle," in IEEE Radar Conference, 2007, pp. 427-430.

[30] Y. Liu, H. Ruan, L. Wang, and A. Nehorai, "The random frequency diverse array: A new antenna structure for uncoupled direction-range indication in active sensing," IEEE Journal of Selected Topics in Signal Processing, vol. 11, no. 2, pp. 295-308, 2016.

[31] S. Qin, Y. D. Zhang, M. G. Amin, and F. Gini, "Frequency diverse coprime arrays with coprime frequency offsets for multitarget localization," IEEE Journal of Selected Topics in Signal Processing, vol. 11, no. 2, pp. 321-335, 2016.

[32] S. M. Kay, Fundamentals of statistical signal processing, Volume I: Estimation theory. Prentice-Hall, Inc., 1993.

[33] S. Sedighi, B. Shankar, M. Soltanalian, and B. Ottersten, "One-bit doa estimation via sparse linear arrays," in IEEE International Conference on Acoustics, Speech, and Signal Processing (ICASSP), 2020. 